\title{
Abundância relativa e riqueza de espécies de aranhas (Arachnida, Araneae) em clareiras originadas da exploração de petróleo na bacia do rio Urucu (Coari, Amazonas, Brasil) Relative abundance and species richness of spiders (Arachnida, Araneae) in forest gaps originated from oil and gas explotation at Urucu River Basin (Coari, Amazonas, Brazil)
}

\author{
Sidclay Calaça Dias' ${ }^{1}$ Alexandre Bragio Bonaldo" \\ Instituto Nacional de Pesquisas da Amazônia. Manaus, Amazonas, Brasil \\ "Museu Paraense Emílio Goeldi/MCTI. Belém, Pará, Brasil
}

\begin{abstract}
Resumo: São investigadas diferenças na abundância relativa e na riqueza de espécies de aranhas em clareiras artificiais com variados níveis de regeneração em Porto Urucu, Coari, Amazonas, Brasil. Foram empregadas três metodologias de coleta complementares, que resultaram na captura de 3.786 indivíduos adultos, identificados em 623 espécies, pertencentes a 39 familias. O presente trabalho foi o que mais acumulou espécies entre todos os artigos publicados com inventários da fauna de aranhas na região neotropical, apesar de $55 \%$ da amostragem terem sido compostos por organismos raros. O algoritmo mais acurado para a presente base de dados, ACE, estimou uma riqueza de 924 espécies. A rarefação feita para cada uma das 33 clareiras amostradas destacou um grupo com poucas espécies acumuladas ( 40 a 43 ) e um grupo com riqueza de espécies significativamente maior (98 a 100) do que as clareiras restantes, apesar das diferenças de número de exemplares e número de espécies entre clareiras não terem sido estatisticamente significativas, indicando que o número de espécies registrado é uma função direta do número de exemplares obtido em cada ponto. Isto sugere que o uso efetivo de dados sobre número de espécies e exemplares de aranhas na avaliação da regeneração de florestas tropicais depende de um esforço amostral muito maior do que o que foi até agora empregado em inventários estruturados destes animais na Amazônia brasileira.
\end{abstract}

Palavras-chave: Diversidade ambiental. Inventário estruturado. Curvas de riqueza. Rarefação. Amazônia.

Abstract: Differences in relative abundance and species richness of spiders in artificial forest gaps with different levels of regeneration at Porto Urucu, Amazonas, Brazil, were investigated. Three complementary collecting methodologies were employed, resulting in the capture of 3.786 adult individuals, belonging to 623 species of 39 spider families. The present study accumulates the highest species number among all other published papers on spider inventories in the Neotropical Region, despite that $55 \%$ of the entire collection was composed of rare organisms. The most accurate algorithm for the present dataset, ACE, estimated a richness of 924 species. Rarefaction curves for each one of the 33 forest gaps sampled highlighted a group of forest gaps which exhibited significantly lower levels of species richness (40 to 43) and another group that exhibited significantly higher levels of species richness (98 to 100). Despite the non-significant differences in abundance and species richness among forest gaps, suggesting that the recorded species number is a direct function of the sampling intensity realized in each site. This suggests that the effective use of data on abundance and species richness of spiders in the evaluation of the regeneration levels of tropical forests depends on a much higher sampling effort than those which have been realized in structured inventories of these animals in Brazilian Amazonia.

Keywords: Environmental diversity. Structured inventory. Richness curves. Rarefaction. Amazon.

DIAS, S. C. \& A. B. BONALDO, 2012. Abundância relativa e riqueza de espécies de aranhas (Arachnida, Araneae) em clareiras originadas da exploração de petróleo na bacia do rio Urucu (Coari, Amazonas, Brasil). Boletim do Museu Paraense Emílio Goeldi. Ciências Naturais 7(2): 123-152.

Autor para correspondência: Sidclay Calaça Dias. Instituto Nacional de Pesquisas da Amazônia. Coordenação de Biodiversidade. Av. André Araújo, 2936, Aleixo. Manaus, AM, Brasil. CEP 69011-970 (sidclaydias@gmail.com).

Recebido em 26/10/2011

Aprovado em 27/07/2012

Responsabilidade editorial: Marinus Hoogmoed

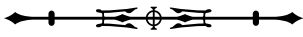




\section{INTRODUÇÃO}

Vários artigos reportam inventários de aranhas como ferramentas usadas para formular e solucionar questões ecológicas relacionadas à dinâmica da fauna no tempo e no espaço, bem como para reconhecer relações diretas entre a fauna e o meio onde elas estão inseridas (Abbott et al., 2003; Azevedo-Ramos et al., 2006; Lo-Man-Hung et al., 2008; Coddington et al., 2009). Mesmo as coletas massivas de aranhas feitas por taxonomistas apresentam informações ecológicas de muita relevância, visto que, após o artigo de Coddington et al. (1991), quase todas as amostragens aracnológicas passaram a ser protocoladas em unidades amostrais. Segundo Coddington et al. (1991) e Gardner et al. (2008), a maximização da informação, obtida por meio do controle do esforço de amostragem com a aplicação de protocolos padronizados, otimiza tempo e dinheiro em expedições ao campo, uma vez que as amostras servem também a propósitos ecológicos.

Diferenças na abundância relativa e na riqueza de espécies de aranhas entre áreas com variadas condições de preservação ambiental foram bem discutidas na literatura (Coyle, 1981; Mclver et al., 1992; Buddle et al., 2000; Willett, 2001; Abbott et al., 2003; Cattin et al., 2003; Baldissera et al., 2004; Larrivée et al., 2005; AzevedoRamos et al., 2006; Lo-Man-Hung et al., 2008; Bonaldo \& Dias, 2010). Para a Amazônia, somente três estudos foram conduzidos nesse sentido: Azevedo-Ramos et al. (2006), que compararam áreas de floresta submetidas a diferentes situações de retirada de madeira no oeste do Pará; LoMan-Hung et al. (2008), que compararam assembleias de aranhas em floresta primárias, secundárias e em áreas de plantação de eucalipto no norte do Pará; e Bonaldo \& Dias (2010), que compararam a fauna de aranhas entre clareiras naturais e artificiais em Porto Urucu, mesmo local onde foi realizado o presente estudo.

Porto Urucu está localizado na Amazônia Central, em uma região com florestas contínuas e bem preservadas, e abriga um campo de exploração de óleo e gás natural desde a década de 1980. Entre os impactos ambientais causados na floresta pela atividade de prospecção e exploração de óleo e gás está a formação de clareiras na matriz florestal. Estas clareiras são abundantes na área de estudo e formam manchas isoladas de desflorestamento, com níveis de regeneração distintos, em meio à matriz de floresta original. Esta situação representa uma boa oportunidade para o estudo da dinâmica das assembleias de aranhas associadas às clareiras. A comparação da riqueza de espécies de aranhas observada entre duas ou mais situações de regeneração ambiental é assunto relativamente comum na literatura (Willett, 2001; Abbott et al., 2003; AzevedoRamos et al., 2006; Lo-Man-Hung et al., 2008). Abbott et al. (2003) encontraram mais indivíduos e espécies de aranhas em ambientes mais impactados do que em locais mais prístinos, comentando que esse resultado pode ser um forte indício da alta resiliência ambiental desses artrópodes. De acordo com esses autores, a abundância relativa e a riqueza de espécies devem cair numericamente após o imediato estabelecimento do impacto antrópico, mas esses parâmetros rapidamente crescem em ordem quantitativa como resultado da disponibilidade de novos microambientes e podem, eventualmente, suplantar números observados em ambientes não impactados.

Apresentam-se os resultados de um inventário estruturado de aranhas coligidas em Porto Urucu, investigando as diferenças na estrutura das assembleias de aranhas, representada pela abundância relativa e riqueza de espécies, em clareiras artificiais, com diferentes idades e tamanhos, e submetidas a intervenções distintas. Buscou-se responder se existem diferenças numéricas consistentes na abundância relativa e na riqueza observada de espécies de aranhas em cada clareira amostrada.

\section{MÉTODOS}

\section{METODOLOGIA DE CAMPO E LABORATÓRIO}

O presente estudo foi conduzido no município de Coari, estado do Amazonas, Brasil. Porto Urucu é uma área pertencente à Petrobras S.A., que lá mantém instalada uma 
estrutura para extração de óleo e gás natural. Localizada na margem direita do rio Urucu, afluente do rio Solimões, Porto Urucu está a $650 \mathrm{~km}$ a oeste de Manaus (4³0' S, $\left.64^{\circ} 30^{\prime} \mathrm{W}\right)$. É uma região coberta principalmente por uma densa floresta ombrófila de terra firme com dossel uniforme, apresentando baixa diversidade de lianas e epífitas (Lima Filho et al., 2001). A fitofisionomia é caracterizada por uniformidade, com poucas áreas inundáveis (igapós e várzeas) ocorrendo nas margens do rio Urucu e seus tributários. As mudanças mais notáveis na estrutura da vegetação ocorrem somente nas áreas com má drenagem do solo ou em clareiras artificiais, abertas com exposição do solo para exploração de óleo e gás natural.

As amostras foram obtidas entre julho e novembro de 2006. Foram feitas coletas em 33 clareiras em estágios de regeneração distintos (Tabela 1). As amostras foram feitas em toda a extensão da clareira, do centro da abertura até $100 \mathrm{~m}$ de floresta adentro, a partir da borda da clareira. Didham (1997), Baldissera et al. (2004) e Rego et al. (2007), que trabalharam com efeito de borda em sistemas florestais sobre fauna de artrópodes, estabeleceram que, em até $100 \mathrm{~m}$ a partir da borda, o efeito é bem pronunciado e reflete melhor os padrões de abundância e riqueza de invertebrados nas clareiras propriamente ditas. Os métodos de captura de aranhas utilizados nesse estudo foram guarda-chuva entomológico, coleta manual noturna e extrator de Winkler (Davies, 1986; Coddington et al., 1991; Brescovit et al., 2004). A coleta manual noturna representa uma fusão dos métodos looking-up e looking-down, propostos originalmente por Coddington et al. (1991). Esta técnica é realizada em um transecto de $30 \mathrm{~m}$, marcado com uma linha, no qual todas as aranhas visualizadas são coletadas durante uma hora, sendo essa unidade de tempo representada como unidade amostral. O guarda-chuva entomológico é uma estrutura de madeira, composta por dois cabos com $1 \mathrm{~m}$, fixados em figuras de $\mathrm{X}$, que sustentam um quadrado de tecido branco de algodão. Esta estrutura é colocada sob pequenos arbustos ou ramos que são agitados com um bastão, de forma a provocar a queda das aranhas sobre o tecido (Davies, 1986). 0 resultado de uma hora de coleta contínua por coletor foi considerado uma amostra. O extrator de Winkler é usado para amostragem de animais ocorrentes na serapilheira. Definiram-se parcelas de $1 \mathrm{~m}^{2}$ no solo da floresta e a serapilheira de cada uma dessas parcelas foi peneirada em um concentrador. $\bigcirc$ material particulado mais os organismos presentes nesse concentrado são segregados com a utilização de extratores de Winkler. Cada amostra de extrator de Winkler correspondeu a $1 \mathrm{~m}^{2}$ de serapilheira peneirada e extraída por 48 horas (Brescovit et al., 2004).

As amostras foram triadas no laboratório de aracnologia do Museu Paraense Emílio Goeldi (MPEG) e todos os espécimes adultos foram depositados na coleção aracnológica desta instituição. Os adultos foram identificados até o nível taxonômico mais exato possível e todos os jovens foram descartados das análises, por não poderem ser identificados com precisão. Nem todos os adultos puderam ser determinados em nível de gênero ou de espécie, mas, por comparação das suas genitálias, pode-se morfotipá-los (separar diferentes espécies por comparação sem que se saiba qual o nome genérico e/ou específico destes indivíduos), garantindo, com precisão, o número de espécies (morfoespécies) deste trabalho. Dezesseis famílias não tiveram nenhum indivíduo identificado até o nível específico.

\section{ANÁLISES ESTATÍSTICAS}

Para comparar eventuais diferenças estatísticas na abundância relativa e na riqueza observada de espécies entre as clareiras, foi usada a análise de variância de um critério (one-way ANOVA), adotando os dados de abundância e riqueza por amostra por clareira (Zar, 1999; Ayres et al., 2007). A transformação dos dados foi feita utilizando o logaritmo na base 10 de cada dado obtido, através do Bioestat 5.0 (Ayres et al., 2007).

Heltshe \& Forrester (1983) desenvolveram um procedimento estatístico para estimar a riqueza de espécies, baseado na presença de espécies únicas nas unidades

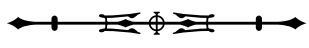


Tabela 1. Coordenada geográfica (CG), abundância e riqueza (número de indivíduos e de espécies de aranhas por clareira) para as 33 clareiras amostradas em Porto Urucu, Coari, Amazonas, Brasil. Os grupamentos destacados são referência aos grupos de clareiras observados na Figura 3.

\begin{tabular}{|c|c|c|c|}
\hline Clareiras & CG & Exemplares & Espécies \\
\hline CL21 & S 4 52' 31"; O $65^{\circ} 10^{\prime} 27^{\prime \prime}$ & 63 & 51 \\
\hline 160 & S $4^{\circ} 48^{\prime} 56^{\prime \prime} ;$ O 65 01' 53" & 54 & 46 \\
\hline 161 & S $4^{\circ} 48^{\prime} 47^{\prime \prime} ;$ O $65^{\circ} 01^{\prime} 57^{\prime \prime}$ & 64 & 50 \\
\hline 170 & S $4^{\circ} 48^{\prime} 23^{\prime \prime} ;$ O $65^{\circ} 02^{\prime} 05^{\prime \prime}$ & 68 & 58 \\
\hline CL26 & S 40 52' 19"; O 65 09' 08" & 99 & 68 \\
\hline J33 & S $4^{\circ} 51^{\prime} 32^{\prime \prime} ;$ O $65^{\circ} 04^{\prime} 44^{\prime \prime}$ & 98 & 74 \\
\hline $\mathrm{j} 43$ & S 4 52' 51"; O 65 08' 56" & 82 & 62 \\
\hline$J 66$ & S $4^{\circ} 48^{\prime} 41^{\prime \prime} ;$ O $65^{\circ} 02^{\prime} 01^{\prime \prime}$ & 85 & 59 \\
\hline 172 & S $4^{\circ} 47^{\prime} 57^{\prime \prime} ;$ O $65^{\circ} 02^{\prime} 14^{\prime \prime}$ & 80 & 57 \\
\hline $\mathrm{LUC3OH}$ & S $4^{\circ} 51^{\prime} 36^{\prime \prime} ;$ O $65^{\circ} 06^{\prime} 23^{\prime \prime}$ & 98 & 73 \\
\hline RUC10 & S $4^{\circ} 52^{\prime} 06^{\prime \prime} ;$ O 65० 15' 52" & 91 & 64 \\
\hline $\mathrm{CL} 1$ & S $4^{\circ} 51^{\prime} 38^{\prime \prime} ;$ O $65^{\circ} 20^{\prime} 04^{\prime \prime}$ & 141 & 95 \\
\hline CL4 & S $4^{\circ} 52^{\prime} 47^{\prime \prime} ;$ O $65^{\circ} 20^{\prime} 09^{\prime \prime}$ & 122 & 80 \\
\hline CL16 & S $4^{\circ} 52^{\prime} 46^{\prime \prime} ;$ O $65^{\circ} 09^{\prime} 50^{\prime \prime}$ & 151 & 95 \\
\hline CL20 & S 4 54' 15"; O 65० 12' 21" & 105 & 80 \\
\hline CL24 & S $4^{\circ} 50^{\prime} 50^{\prime \prime} ; 065^{\circ} 05^{\prime} 03^{\prime \prime}$ & 148 & 102 \\
\hline CL28 & S $4^{\circ}$ 50' 01"; O $65^{\circ} 03^{\prime}$ 53" & 140 & 92 \\
\hline 122 & S 40 53' 14"; O $65^{\circ} 13^{\prime} 37^{\prime \prime}$ & 133 & 89 \\
\hline$J 25$ & S $4^{\circ} 53^{\prime} 10^{\prime \prime} ;$ O $65^{\circ} 11^{\prime} 24^{\prime \prime}$ & 124 & 93 \\
\hline J34 & S $4^{\circ}$ 51' 27"; O 65 04' 46" & 120 & 85 \\
\hline J36 & 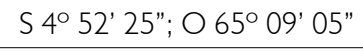 & 134 & 96 \\
\hline J48 & S 40 52' 11"; O 65 $08^{\prime} 05^{\prime \prime}$ & 146 & 101 \\
\hline J52 & S $4^{\circ} 50^{\prime} 32^{\prime \prime} ;$ O $65^{\circ} 04^{\prime} 80^{\prime \prime}$ & 142 & 87 \\
\hline $\mathrm{J} 57$ & S $4^{\circ}$ 45' 47"; O 65० 02' 41" & 121 & 77 \\
\hline J59 & S $4^{\circ} 48^{\prime} 56^{\prime \prime} ;$ O $65^{\circ} 01^{\prime} 53^{\prime \prime}$ & 109 & 82 \\
\hline $\mathrm{J} 63$ & S $4^{\circ} 48^{\prime} 45^{\prime \prime} ;$ O $65^{\circ} 01^{\prime} 58^{\prime \prime}$ & 103 & 78 \\
\hline 173 & S 4 $51^{\prime} 35^{\prime \prime} ;$ O $65^{\circ} 06^{\prime} 17^{\prime \prime}$ & 130 & 88 \\
\hline $\mathrm{J} 74$ & S $4^{\circ}$ 51' 26"; O 65 06' 06" & 130 & 90 \\
\hline$J 77$ & S 40 53' 45"; O $65^{\circ} 19^{\prime} 11^{\prime \prime}$ & 118 & 89 \\
\hline 187 & S $4^{\circ}$ 50’ 30"; O 65 03' 51" & 132 & 80 \\
\hline RUC7 & S 4 $4^{\circ} 54^{\prime} 16^{\prime \prime} ;$ O $65^{\circ} 19^{\prime} 37^{\prime \prime}$ & 112 & 73 \\
\hline $\mathrm{CL3}$ & S $4^{\circ} 51^{\prime} 54^{\prime \prime} ; 065^{\circ} 20^{\prime} 02^{\prime \prime}$ & 185 & 103 \\
\hline 12 & S $4^{\circ} 53^{\prime} 30^{\prime \prime} ; O 65^{\circ} 20^{\prime} 07^{\prime \prime}$ & 158 & 105 \\
\hline
\end{tabular}

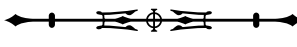


amostrais. Este procedimento é utilizado para reduzir subestimativas dos valores reais de uma dada espécie em uma assembleia, com base no número de organismos raros (Colwell \& Coddington, 1994). Os estimadores produzidos podem ser ajustados e visualizados em uma curva plotada no mesmo tipo de gráfico de esforço amostral (riqueza de espécies na abscissa e amostras na ordenada). Por meio do programa EstimateS 8.0.0 (Colwell, 2005), foram calculados todos os estimadores não paramétricos disponíveis para a totalidade das espécies observadas no inventário. Gotelli \& Colwell (2001) estabeleceram que os dados sobre a riqueza de espécies plotados contra o número de indivíduos (densidade de espécies) é o meio mais acurado para se produzir curvas de rarefação, usando-se intervalos de confiança dos dados da riqueza observada. Utilizou-se o programa EcoSim 7.72 (Gotelli \& Entsminger, 2005) para se calcular a rarefação para cada uma das 33 clareiras, adotando-se os dados primários de abundância com base no número de indivíduos. $O$ algoritmo de randomização usado foi a própria curva de rarefação e a unidade de esforço foi o indivíduo (Gotelli \& Colwell, 2001; Gotelli \& Entsminger, 2005). Segundo Gotelli \& Colwell (2001), nos resultados das rarefações, os valores do número de amostras são diluídos estatisticamente, o que permite a comparação de áreas amostradas com esforços diferentes. Outra vantagem óbvia das curvas de rarefação é a possibilidade do cálculo dos seus respectivos intervalos de confiança de 95\%. Estes intervalos foram plotados para dois grupos de curvas que representam os menores e os maiores valores de riqueza observada, o que permitiu discernir padrões significativamente diferentes de riqueza de espécies em algumas clareiras. A intensidade amostral (IA) foi definida como a proporção de adultos por espécies (Coddington et al., 1996).

\section{RESULTADOS E DISCUSSÃO}

De 626 amostras válidas para as análises, um total de 3.786 adultos foi coletado em 33 clareiras com diferentes estágios de regeneração, representando 623 espécies em 39 famílias. Drymusidae e Hersilidae foram, cada uma, representadas somente por um único indivíduo adulto. Como em outros inventários Neotropicais (Borges \& Brescovit, 1996; Silva \& Coddington, 1996; Ricetti \& Bonaldo, 2008), as famílias mais abundantes e ricas nesse estudo foram Theridiidae (880 indivíduos; 117 morfoespécies), Araneidae (841 indivíduos; 103 morfoespécies) e Salticidae (433 indivíduos; 81 morfoespécies). Do total de espécies coletadas, 248 foram singletons e 98 foram doubletons. A Tabela 2 mostra os resultados da análise de variância de um fator, evidenciando os respectivos resultados para abundância e riqueza de aranhas amostradas em 33 clareiras. A Tabela 3 mostra o número de indivíduos e espécies por família coligida em Porto Urucu em todas as clareiras, com as abundâncias por metodologia de coleta e o Apêndice traz a lista completa de espécies e morfoespécies registradas nas amostras, com a abundância total de cada uma.

O presente trabalho foi o que mais acumulou espécies entre os artigos publicados com inventários rápidos da fauna de aranhas na região neotropical (Borges \& Brescovit, 1996 - 102 espécies observadas; Silva \& Coddington, 1996 - 498; Dias et al., 2006 - 166; Bonaldo et al., 2007 - 212; Ricetti \& Bonaldo, 2008 - 427; Bonaldo \& Dias, 2010 - 393). As diferenças encontradas entre a riqueza observada de espécies em inventários feitos em locais diferentes podem ser reflexo de diferenças na complexidade ambiental entre os habitat amostrados e/ou de diferenças de esforço amostral em termos de quantidades de amostras produzidas ou metodologias

Tabela 2. Resultados da análise de variância de um fator, mostrando os respectivos resultados para abundância e riqueza de aranhas amostradas em 33 clareiras em Porto Urucu, Coari, Amazonas, Brasil. GL = grau de liberdade, SQ = soma dos quadrados, QM = quadrado médio.

\begin{tabular}{c|c|c|c|c|c|c}
\hline Fontes de variação & GL (abundância) & SQ (abundância) & QM (abundância) & GL (riqueza) & SQ (riqueza) & QM (riqueza) \\
\hline Tratamentos & 32 & 97,1 & 30,331 & 32 & 90,8 & 28,383 \\
\hline Erro & 593 & 12,5 & 21,005 & 594 & 11,7 & 19,638 \\
\hline
\end{tabular}

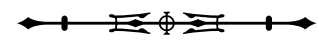


Tabela 3. Abundância total de exemplares e riqueza total de espécies por família de aranhas coligidas em Porto Urucu, Coari, Amazonas, Brasil, em 33 clareiras. GCE = guarda-chuva entomológico, $\mathrm{N}=$ coleta manual noturna, Win = coleta com extrator de Winkler.

(Continua)

\begin{tabular}{|c|c|c|c|c|c|}
\hline Famílias & $\begin{array}{l}\text { Número de } \\
\text { exemplares }\end{array}$ & Espécies & GCE & N & Win \\
\hline Anapidae & 23 & 4 & 0 & 0 & 23 \\
\hline Anyphaenidae & 36 & 11 & 26 & 10 & 0 \\
\hline Araneidae & 841 & 103 & 403 & 429 & 9 \\
\hline Caponiidae & 3 & 1 & 3 & 0 & 0 \\
\hline Clubionidae & 7 & 2 & 7 & 0 & 0 \\
\hline Corinnidae & 157 & 44 & 72 & 70 & 15 \\
\hline Ctenidae & 234 & 22 & 5 & 229 & 0 \\
\hline Deinopidae & 24 & 1 & 2 & 22 & 0 \\
\hline Dipluridae & 6 & 3 & 0 & 6 & 0 \\
\hline Drymusidae & 1 & 1 & 0 & 1 & 0 \\
\hline Gnaphosidae & 8 & 3 & 5 & 1 & 2 \\
\hline Hahniidae & 4 & 1 & 0 & 0 & 4 \\
\hline Hersiilidae & 1 & 1 & 0 & 1 & 0 \\
\hline Hersiliidae & 5 & 1 & 0 & 5 & 0 \\
\hline Linyphiidae & 93 & 14 & 2 & 2 & 89 \\
\hline Lycosidae & 2 & 2 & 0 & 2 & 0 \\
\hline Mimetidae & 36 & 11 & 21 & 15 & 0 \\
\hline Mysmenidae & 3 & 3 & 0 & 0 & 3 \\
\hline Nephilidae & 4 & 1 & 0 & 4 & 0 \\
\hline Oonopidae & 53 & 21 & 9 & 4 & 40 \\
\hline Oxyopidae & 95 & 7 & 95 & 0 & 0 \\
\hline Pholcidae & 182 & 11 & 84 & 97 & 1 \\
\hline Pisauridae & 36 & 5 & 24 & 12 & 0 \\
\hline Salticidae & 433 & 81 & 333 & 53 & 47 \\
\hline Scytodidae & 52 & 2 & 33 & 19 & 0 \\
\hline Segestriidae & 2 & 2 & 2 & 0 & 0 \\
\hline Selenopidae & 8 & 7 & 5 & 3 & 0 \\
\hline Senoculidae & 14 & 3 & 9 & 5 & 0 \\
\hline Sparassidae & 42 & 14 & 10 & 32 & 0 \\
\hline Symphytognathidae & 3 & 3 & 0 & 0 & 3 \\
\hline Synotaxidae & 4 & 3 & 4 & 0 & 0 \\
\hline Tetragnathidae & 61 & 20 & 30 & 31 & 0 \\
\hline Theraphosidae & 7 & 5 & 0 & 7 & 0 \\
\hline Theridiidae & 880 & 117 & 521 & 332 & 27 \\
\hline
\end{tabular}


Tabela 3.

(Conclusão)

\begin{tabular}{c|c|c|c|c|c}
\hline Famílias & $\begin{array}{c}\text { Número de } \\
\text { exemplares }\end{array}$ & Espécies & GCE & W & 31 \\
\hline Theridiosomatidae & 87 & 18 & 51 & 14 & 5 \\
\hline Thomisidae & 123 & 30 & 105 & 9 & 71 \\
\hline Trechaleidae & 37 & 7 & 98 & 1 & 0 \\
\hline Uloboridae & 169 & 36 & 1 & 1.537 & 8 \\
\hline Zodariidae & 10 & 623 & 1.969 & 280 \\
\hline Total & 3.786 & & & \\
\hline
\end{tabular}

empregadas. Uma consequência dessas diferenças pode ser o acréscimo de espécies ao elenco já estabelecido de uma dada área de estudo. Comparando-se a lista de espécies determinadas ao nível específico, fornecida por Bonaldo \& Dias (2010), com a produzida neste trabalho, ambas advindas de Porto Urucu, observa-se um incremento de 31\% de espécies identificadas. Este acréscimo é devido, em parte, ao maior refinamento taxonômico obtido no presente trabalho, mas também é principalmente um reflexo da maior quantidade de amostras produzidas. A resolução taxonômica em táxons megadiversos é um fator limitante nas análises faunísticas em regiões tropicais, pois a maioria dos organismos coletados pertence a grupos carentes de revisões taxonômicas (Bonaldo et al., 2009). Cerca de 23\% das espécies coletadas neste estudo foram determinadas até o nível específico (144 das 623 espécies coligidas). As demais espécies foram determinadas em nível de morfoespécie (ou unidade taxonômica operacional UTO, de acordo com Oliver \& Beattie, 1996). As famílias mais abundantes neste estudo, Araneidae e Theridiidae, foram as que tiveram melhor resolução taxonômica (55 e 32 espécies identificadas, respectivamente).

A Tabela 4 indica a intensidade amostral por clareira, os valores observados de singletons e percentagem de singletons. A intensidade amostral (IA) total observada neste trabalho foi de 6,1. Esse valor é baixo se comparado aos inventários estruturados realizados na América do Norte (e.g. Coddington et al., 1996, Appalachian Mountains, IA = 18,3) e na Europa (e.g. Scharffet al., 2003, Dinamarca, IA =
132). Entretanto, valores baixos de intensidade amostral são comuns em inventários conduzidos em áreas tropicais, como observado em Coddington et al. (1991) (três localidades na Bolívia, IA < 5), Bonaldo et al. (2007) (Rio Grande do Sul, Brasil; IA = 8,8), Ricetti \& Bonaldo (2008) (Serra do Cachimbo, Pará; IA = 6,44) e Bonaldo \& Dias (2010) (Porto Urucu, Amazonas; IA = 4,1). A baixa intensidade amostral é indicativa de que o inventário não está completo e de que há necessidade de mais esforço para que se diminua a quantidade de singletons (Coddington et al., 2009). A intensidade amostral por clareira é ainda mais baixa, razão pela qual não foram produzidas curvas de acúmulo e de estimativas de riqueza de espécies para cada uma das 33 clareiras amostradas. A Figura 1 mostra que, apesar de as diferenças de abundância e riqueza entre clareiras não terem sido estatisticamente significativas, existe uma relação detectável entre abundância e riqueza em cada clareira, ou seja, quanto maior o esforço e, em menor medida, a intensidade amostral obtida em uma dada clareira, maior será o número registrado de espécies. Para melhor visualização, foram retiradas da Figura 2 as curvas que estavam sobrepostas umas às outras, uma vez que os respectivos valores são muito semelhantes (uniques está sobreposta com a curva de singletons, duplicates está sobreposta com a curva de doubletons, e as curvas de 'Chao 1', 'Chao 2' e 'ICE' estão sobrepostas com a curva de 'ACE'). A amplitude da variação da intensidade amostral entre as 33 clareiras foi pequena e esta variação explica apenas parcialmente as variações de riqueza e abundância observadas. Isto se 
Tabela 4. Número de amostras, intensidade amostral, de singletons e percentagem de singletons por clareira amostrada em Porto Urucu, Coari, Amazonas, Brasil.

\begin{tabular}{|c|c|c|c|c|}
\hline Clareiras & Número de amostras & Intensidade amostral & Singletons & Percentagem de singletons \\
\hline CL1 & 17 & 1,48 & 75 & $80 \%$ \\
\hline CL16 & 20 & 1,6 & 62 & $65 \%$ \\
\hline CL20 & 15 & 1,31 & 62 & $77,5 \%$ \\
\hline CL21 & 16 & 1,24 & 42 & $82 \%$ \\
\hline CL24 & 22 & 1,45 & 76 & $74,5 \%$ \\
\hline CL26 & 17 & 1,46 & 50 & $73,5 \%$ \\
\hline CL28 & 23 & 1,52 & 65 & $71 \%$ \\
\hline CL3 & 23 & 1,8 & 68 & $66 \%$ \\
\hline CL4 & 17 & 1,53 & 63 & $79 \%$ \\
\hline 12 & 20 & 1,5 & 75 & $71 \%$ \\
\hline 122 & 23 & 1,38 & 69 & $77,5 \%$ \\
\hline 125 & 20 & 1,35 & 76 & $83 \%$ \\
\hline J33 & 18 & 1,32 & 62 & $84 \%$ \\
\hline J34 & 15 & 1,41 & 72 & $85 \%$ \\
\hline 136 & 17 & 1,4 & 78 & $81 \%$ \\
\hline J43 & 15 & 1,32 & 51 & $82 \%$ \\
\hline j48 & 21 & 1,45 & 75 & $74 \%$ \\
\hline 152 & 21 & 1,63 & 57 & $65,5 \%$ \\
\hline J57 & 19 & 1,55 & 55 & $70,5 \%$ \\
\hline J59 & 19 & 1,33 & 66 & $84,5 \%$ \\
\hline 160 & 16 & 1,17 & 40 & $87 \%$ \\
\hline$J 61$ & 16 & 1,28 & 40 & $80 \%$ \\
\hline 163 & 20 & 1,32 & 64 & $82 \%$ \\
\hline 166 & 19 & 1,47 & 43 & $74 \%$ \\
\hline 170 & 17 & 1,17 & 53 & $91 \%$ \\
\hline 172 & 14 & 1,4 & 42 & $74 \%$ \\
\hline 173 & 24 & 1,48 & 64 & $73 \%$ \\
\hline 174 & 24 & 1,44 & 74 & $82 \%$ \\
\hline 177 & 19 & 1,33 & 73 & $82 \%$ \\
\hline 187 & 20 & 1,68 & 54 & $68 \%$ \\
\hline $\mathrm{LUC30H}$ & 21 & 1,34 & 58 & $79 \%$ \\
\hline RUC10 & 17 & 1,42 & 50 & $78 \%$ \\
\hline RUC7 & 21 & 1,53 & 55 & $75 \%$ \\
\hline
\end{tabular}




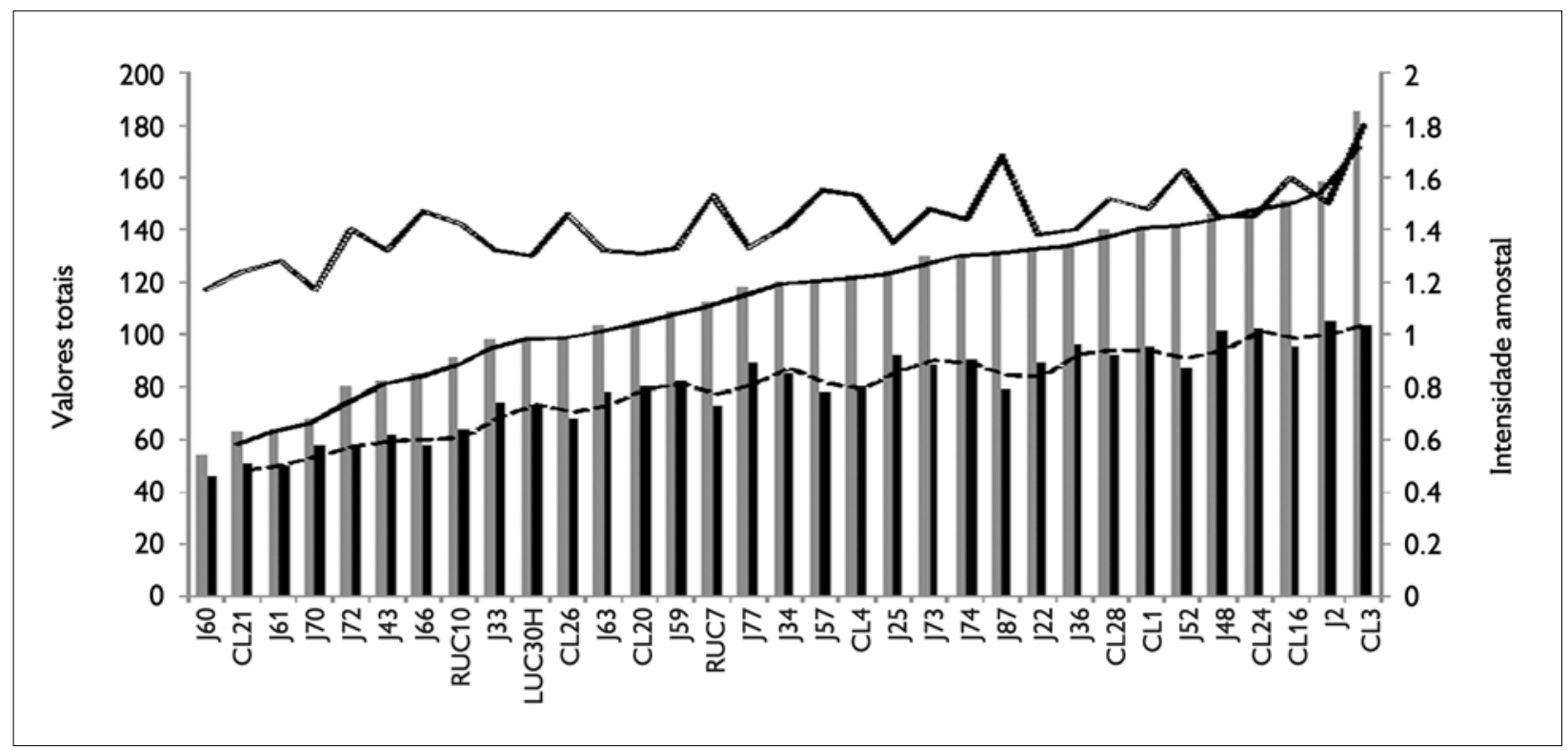

Figura 1. Valores de abundância, riqueza e intensidade amostral das 33 clareiras amostradas em Porto Urucu, Coari, Amazonas, Brasil, ordenados por abundância. As barras cinzas e a linha cheia representam os valores de abundância, as barras negras e a linha pontilhada representam os valores de riqueza. A linha vazada representa os valores de intensidade amostral obtidos em cada clareira.

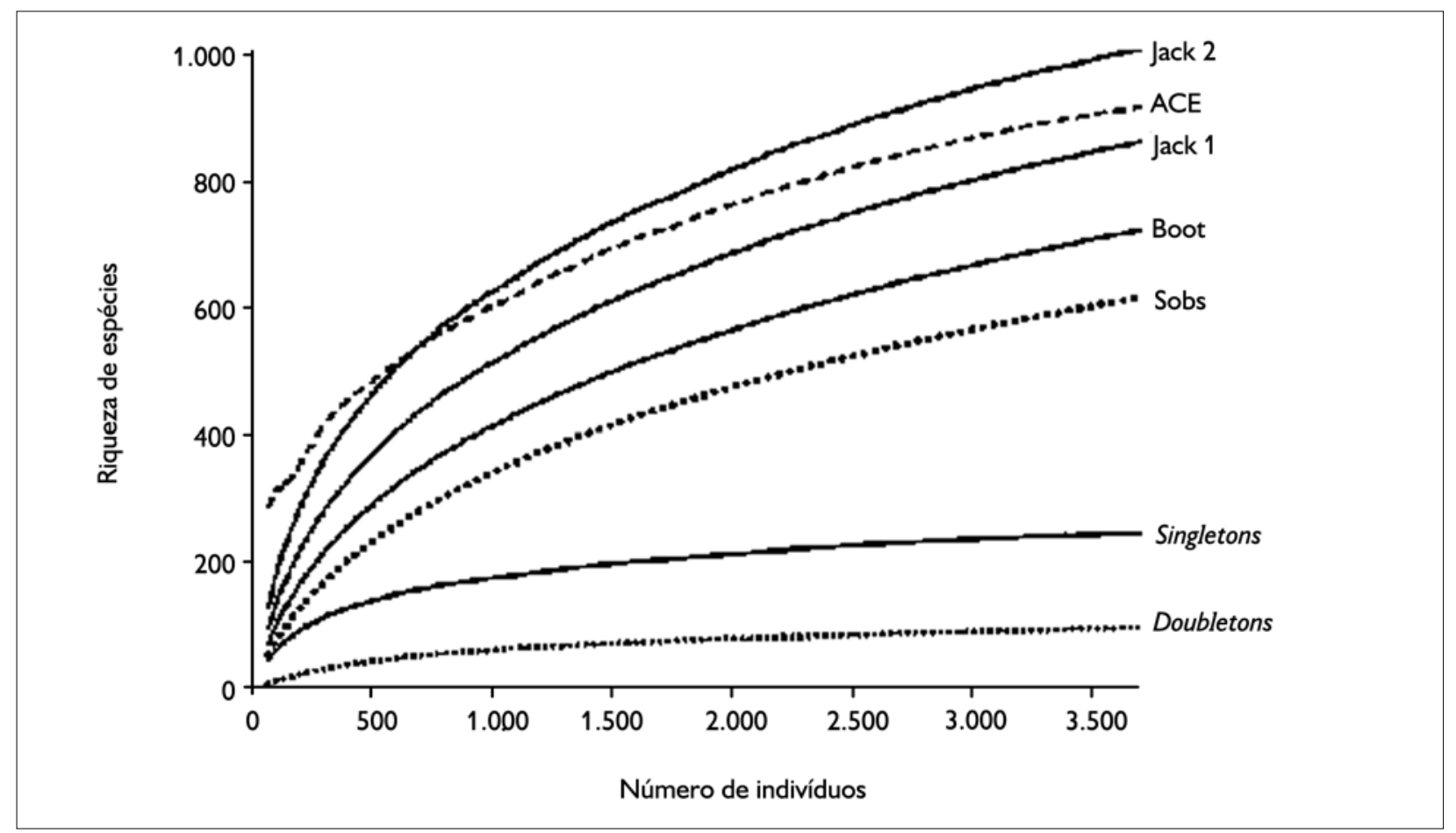

Figura 2. Curvas observadas e estimadas de acúmulo de espécies, baseadas no número de indivíduos coligidos. Fauna de aranhas de Porto Urucu, Coari, Amazonas, Brasil. Espécies observadas (Species observed - Sobs) = 623; Estimador de Cobertura de Abundância (Abundance Coverage Estimator $-\mathrm{ACE})=924 ;$ Singletons $=248$

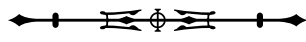


deve, provavelmente, à eficiência diferencial dos métodos de coleta empregados, cujo número de amostras válidas diferiu de clareira para clareira.

As análises de estimativas de espécies feitas para toda a fauna coletada nas clareiras artificiais em Porto Urucu mostraram que, apesar do número de espécies observadas (Sobs = 623) não ter atingido sua assíntota, o estimador não paramétrico que tendeu mais fortemente à estabilização foi ACE, com 924 espécies acumuladas (Figura 2). Este estimador indica que, para completar o inventário de aranhas em Porto Urucu, necessita-se acrescentar à Tabela 3 no mínimo mais 301 espécies de aranhas que não sejam raras. As estimativas de riqueza permitem acessar a completitude de um inventário (Coddington et al., 1996). Contudo, em ecossistemas tropicais, onde ocorre grande número de singletons e as intensidades amostrais são baixas, as estimativas de riqueza total, obtidas por meio de curvas de acumulação baseadas na incidência e abundância de espécies raras, devem ser avaliadas com cuidado, pois a confidência dos resultados é uma função do sucesso na obtenção de taxas altas de representatividade amostral (Coddington et al., 2009). Ainda assim, o uso de curvas de acúmulo de espécies baseadas em organismos raros ou pouco incidentes é uma ferramenta válida para auxiliar a execução de inventários de táxons megadiversos nos trópicos, uma vez que fornecem estimativas quantitativas cuja precisão pode ser aprimorada com o aumento de esforço e a consequente diminuição da incidência de singletons, em situações de inventários de longo prazo. Neste estudo, a quantidade de singletons supera a expectativa de Coddington et al. (2009), que é de cerca de 32\% do número de espécies representadas por um único indivíduo adulto em inventários de comunidades tropicais. Segundo estes autores, a provável causa para a grande percentagem de espécies raras em ecossistemas tropicais é a subamostragem, que determina uma tendência positiva nas estimativas de riqueza real de espécies. Coddington et al. (2009) propõem reduzir o efeito da subamostragem pela simples ampliação do esforço, de forma a acrescentar mais indivíduos por espécies no inventário. Assim, o uso de dados de inventários faunísticos de grupos megadiversos na caracterização de processos ecológicos em ecossistemas tropicais, onde estes inventários nunca são exaustivos, depende de um esforço amostral muito maior do que o que foi até agora empregado em inventários estruturados na Amazônia brasileira.

Os resultados da rarefação mostraram que 27 das clareiras amostradas não formam grupos claros, significativamente diferentes entre si, em relação à riqueza de espécies (Figura 3). Estas clareiras apresentaram riquezas de espécies intermediárias e os intervalos de confiança de suas curvas de rarefação estão sobrepostos sequencialmente. Entretanto, as clareiras restantes formam dois grupos, com riqueza de espécies extremas, significativamente diferentes: um grupo de quatro clareiras (CL21, J60, J61 e J70) com riquezas de espécies estatisticamente iguais entre si, mas significativamente menores do que a apresentada por todas as outras clareiras; e um grupo formado por duas clareiras (CL3 e 12), com riquezas de espécies estatisticamente iguais entre si, mas significativamente maiores do que a apresentada por todas as outras clareiras. A clareira CL16 apresentou valores de riqueza de espécies apenas um pouco menor do que os apresentados pela clareira CL3, que pertence ao grupo com maior riqueza. Entretanto, o intervalo de confiança da curva de rarefação daquela clareira (não mostrado no gráfico da Figura 2) não se sobrepõe ao intervalo da curva da clareira CL3 ao final da adição de indivíduos. $\bigcirc$ esforço amostral despendido em algumas clareiras não foi suficiente para refletir diferenças estatísticas entre a abundância e a riqueza das aranhas. Os dois grupos de clareiras com riquezas de espécies extremas não são artefatos, pois foram definidos estatisticamente por meio de intervalos de confiança de curvas de rarefação.

Vários estudos, em diferentes regiões, apontam que a tendência geral para a fauna de aranhas é que a abundância relativa e a riqueza de espécies sejam diferentes em áreas com diferentes níveis ou graus de regeneração (Coyle, 1981; Mclver et al., 1992; Buddle et al., 2000; Willett, 2001; Larrivée et al., 2005; Azevedo-Ramos et al., 


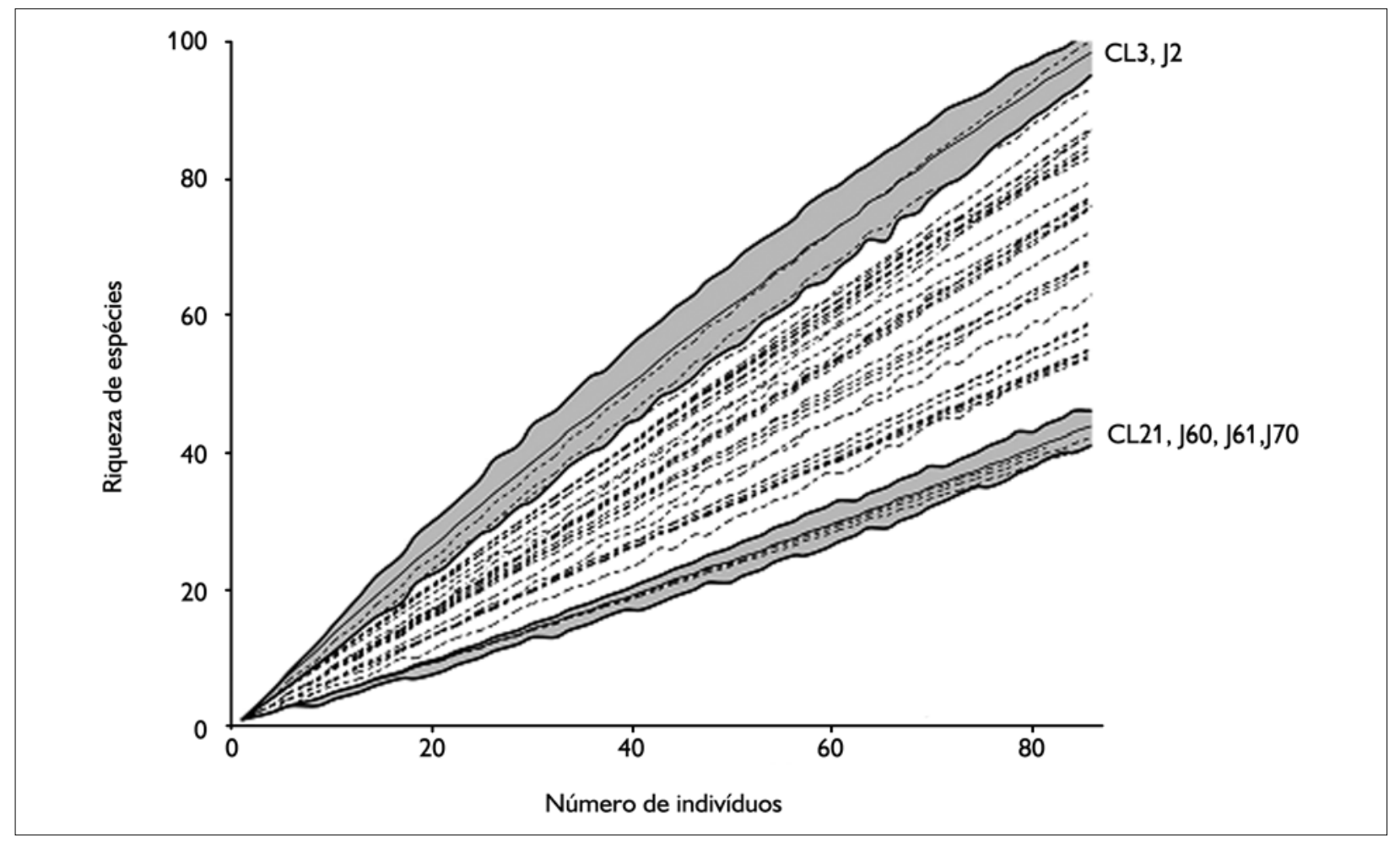

Figura 3. Curvas de rarefação das 33 clareiras com base no número de indivíduos coletados em Porto Urucu, Coari, Amazonas, Brasil. As áreas sombreadas representam dois grupamentos de clareiras delimitados por intervalos de confiança de $95 \%$.

2006; Lo-Man-Hung et al., 2008). Azevedo-Ramos et al. (2006) e Lo-Man-Hung et al. (2008) apontaram diferenças nas abundâncias relativas das aranhas coletadas em variadas situações de reflorestamento, indicando que a abundância relativa de exemplares em áreas impactadas é maior que em lugares que não sofreram impactos. Abbott et al. (2003) citam alguns motivos de não terem sido registradas diferenças entre a abundância de exemplares e a riqueza de espécies, observadas entre áreas com distintas condições de regeneração. Segundo esses autores, a principal razão para a ausência dessa diferença seria a alta resiliência das aranhas, que faz com que a fauna seja similar, independente do estado do ambiente onde se realize a coleta. É possível que, dada a sua alta plasticidade ambiental, a fauna de aranhas possa se recompor rapidamente em clareiras que sofreram intervenções recentes, tornando-se equiparável às clareiras mais antigas em termos de abundância relativa e riqueza de espécies. A falta de distinção da abundância e riqueza de aranhas entre os locais de amostragem pode ser decorrente da falta de características que diferenciem as clareiras amostradas para refletir uma diferença real na abundância e na riqueza das aranhas na área de estudo. Recomenda-se que medidas fitossociológicas, por exemplo, diâmetro na altura do peito, altura das árvores, abertura do dossel e complexidade estrutural da vegetação (Brokaw, 1985; Schnitzer \& Carson, 2001), sejam tomadas em futuros estudos como forma de se estabelecer níveis precisos de regeneração que possam vir a explicar eventuais mudanças na comunidade de aranhas. $\mathrm{O}$ uso de grupamentos ecológicos especializados de aranhas (guildas) e/ou táxons ecologicamente mais distintos que outros pode também ser uma alternativa para estudos futuros, no sentido de estabelecer gradientes de regeneração das clareiras originadas pela exploração petrolífera.

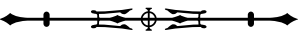




\section{AGRADECIMENTOS}

Ao CNPq pela bolsa de estudos concedida a SCD e de produtividade em pesquisa (\#303591/2006-3 - ABB); à Rede CTPetro Amazônia - Fundo Setorial do Petróleo (Financiadora de Estudos e Projetos/Conselho Nacional de Desenvolvimento Científico e Tecnológico/PETROBRAS), pelo financiamento das expedições ao campo e pela disponibilização de equipamentos e material de consumo necessários à execução deste trabalho. Parte do equipamento de laboratório utilizado foi proporcionada pelo Programa de Pesquisa em Biodiversidade - Amazônia Oriental (PPBio).

\section{REFERÊNCIAS}

ABBOTT, I., T. BURBIDGE, K. STREHLOW, A. MELLICAN \& A. WILLS, 2003. Logging and burning impacts on cockroaches, crickets, and spiders in Jarrah forest Western Australia. Forest Ecology and Management 174(1-3): 383-399.

AYRES, M., M. AYRES JR., D. L. AYRES \& A. S. SANTOS, 2007. BioEstat 5.0: aplicações estatísticas nas áreas das ciências biológicas e médicas: 1-364. Sociedade Civil Mamirauá, Belém.

AZEVEDO-RAMOS, C., O. CARVALHO JR. \& B. D. AMARAL, 2006. Short-term effects of reduced-impact logging on eastern Amazon fauna. Forest Ecology and Management 232 (1-3): 26-35.

BALDISSERA, R., G. GANADE \& S. B. FONTOURA, 2004. Web spider community response along an edge between pasture and Araucaria forest. Biological Conservation 118(3): 403-409.

BONALDO, A. B., M. A. L. MARQUES, R. PINTO-DA-ROCHA \& T. A. GARDNER, 2007. Species richness and community structure of arboreal spider assemblages in fragments of three forest types at Banhado Grande wet plain, Gravataí River, Rio Grande do Sul, Brazil. Iheringia, Série Zoologia 97(2): 143-151.

BONALDO, A. B., A. D. BRESCOVIT, H. HÖFER, T. R. GASNIER \& A. A. LISE, 2009. A araneofauna (Arachnida, Araneae) da Reserva Florestal Ducke, Manaus, Amazonas, Brasil. In: C. R. V. FONSECA, C. MAGALHÃES, J. A. RAFAEL \& E. N. FRANKLIN (Eds.): A Fauna de artrópodes da Reserva Florestal Ducke: estado atual do conhecimento taxonômico e biológico: 201-222. Editora INPA, Manaus.

BONALDO, A. B. \& S. C. DIAS, 2010. A structured inventory of spiders (Arachnida, Araneae) in natural and artificial forest gaps at Porto Urucu, Western Brazilian Amazonia. Acta Amazonica 40(2): 357-372.

BORGES, S. H. \& A. D. BRESCOVIT, 1996. Inventário preliminar da aracnofauna (Araneae) de duas localidades na Amazônia Ocidental. Boletim do Museu Paraense Emílio Goeldi, Série Zoologia 12(1): 9-21.
BRESCOVIT, A. D., R. BERTANI, R. PINTO-DA-ROCHA \& C. A. RHEIMS, 2004. Aracnídeos da Estação Ecológica Juréia-Itatins: inventário preliminar e história natural. In: O. A. V. MARQUES \& W. DULEBA (Eds.): Estação Ecológica Juréia-Itatins: ambiente físico, flora e fauna: 198-221. Holos, Ribeirão Preto.

BROKAW, N. V. L., 1985. Gap-phase regeneration in a tropical forest. Ecology 66(3): 682-687.

BUDDLE, C. M., J. R. SPENCE \& D. W. LANGNOR, 2000. Succession of boreal forest spider assemblages following wildfire and harvesting. Ecography 23(4): 424-436.

CATTIN, M.-F., G. BLANDENIER, C. BANAŠEK-RICHTER \& L.-F. BERSIER, 2003. The impact of mowing as a management strategy for wet meadows on spider (Araneae) communities. Biological Conservation 113(2): 179-188.

CODDINGTON, J. A., C. E. GRISWOLD, D. SILVA, D. PEÑARANDA \& S. LARCHER, 1991. Designing and testing sampling protocols to estimate biodiversity in tropical ecosystems. In: E. C. DUDLEY (Ed.): The unity of evolutionary biology. Proceedings of The International Congress of Systematic and Evolutionary Biology 4: 44-60.

CODDINGTON, J. A., L. H. YOUNG \& F. A. COYLE, 1996. Estimating spider species richness in a southern Appalachian cove hardwood forest. Journal of Arachnology 24: 111-128.

CODDINGTON, J. A., I. AGNARSSON, J. A. MILLER, M. KUNTNER \& G. HORMIGA, 2009. Undersampling bias: the null hypothesis for singleton species in tropical arthropod surveys. Journal of Animal Ecology 78(3): 573-584.

COLWELL, R. K., 2005. EstimateS: estimation of species richness and shared species from samples. Version 8.0. Disponível em: <http:// viceroy.eeb.uconn.edu/EstimateS>. Acesso em: 20 dezembro 2009.

COLWELL, R .K. \& J. A. CODDINGTON, 1994. Estimating terrestrial biodiversity through extrapolation. Philosophical Transactions of the Royal Society of London, Series B 345: 101-118.

COYLE, F. A., 1981. Effects of clearcutting on the spider community of a Southern Appalachian forest. Journal of Arachnology 9: 285-298.

DAVIES, V. T., 1986. Australian spiders (Araneae): collection, preservation and identification: 1-60. Queensland Museum (Queensland Museum Booklet, 14), Brisbane.

DIAS, S. C., A. D. BRESCOVIT, E. C. COUTO \& C. F. MARTINS, 2006. Species richness and seasonality of spiders (Arachnida, Araneae) in an urban Atlantic Forest fragment in Northeastern Brazil. Urban Ecosystems 9(4): 323-335.

DIDHAM, R. K., 1997. The influence of edge effects and forest fragmentation on leaf litter invertebrates in Central Amazonia. In: W. F. LAURENCE \& R. O. BIERREGAARD JR. (Eds.): Tropical forest remnants: ecology, management, and conservation of fragmented communities: 55-70. University of Chicago Press, Chicago. 
GARDNER, T. A., J. BARLOW, I. S. ARAÚJO, T. C. ÁVILA-PIRES, A. B. BONALDO, J. E. COSTA, M. C. ESPÓSITO, L. V. FERREIRA, J. HAWES, M. I. M. HERNANDEZ, M. S. HOOGMOED, R. N. LEITE, N. F. LO-MAN-HUNG, J. R. MALCOLM, M. B. MARTINS, L. A. M. MESTRE, R. MIRANDA-SANTOS, W. L. OVERAL, L. PARRY, S. L. PETERS, M. A. RIBEIRO-JUNIOR, M. N. F. DA SILVA, C. S. MOTTA \& C. A. PERES, 2008. The cost-effectiveness of biodiversity surveys in tropical forests. Ecology Letters 11(2): 139-150.

GOTELLI, N. J. \& G. L. ENTSMINGER, 2005. EcoSim: null models software for ecology. Version 7.0. Acquired Intelligence Inc. \& KeseyBear. Disponível em: <http://homepages.together.net/ gentsmin/ ecosim.htm>. Acesso em: 20 dezembro 2009.

GOTELLI, N. J. \& R. K. COLWELL, 2001. Quantifying biodiversity: procedures and pitfalls in the measurement and comparison of species richness. Ecology Letters 4(4): 379-391.

HELTSHE, J. F. \& N. E. FORRESTER, 1983. Estimating species richness using the jackknife procedure. Biometrics 39(1): 1-11.

LARRIVÉE, M., L. FAHRIG \& P. DRAPEAU, 2005. Effects of a recent wildfire and clearcuts on ground-dwelling boreal forest spider assemblages. Canadian Journal of Forest Research 35(11): 2575-2588.

LIMA FILHO, D. A., F. D. A. MATOS, I. L. AMARAL, J. REVILLA, L. S. COÊLHO, J. F. RAMOS \& J. L. SANTOS, 2001. Inventário florístico de floresta ombrófila densa de terra firme, na região do Rio Urucu - Amazonas, Brasil. Acta Amazonica 31(4): 565-579.

LO-MAN-HUNG, N. F., T. A. GARDNER, M. A. RIBEIRO-JÚNIOR, J. BARLOW \& A. B. BONALDO, 2008. The value of primary, secondary, and plantation forests for Neotropical epigeic arachnids. Journal of Arachnology 36(2): 394-401.

MCIVER, J. D., G. L. PARSONS \& A. R. MOLDENKE, 1992. Litter spider succession after clear-cutting in a western coniferous forest. Canadian Journal of Forest Research 22: 984-992.
OLIVER, I. \& A. J. BEATTIE, 1996. Invertebrate morphospecies as surrogate for species: a case study. Conservation Biology 10(1): 99-109.

REGO, F. N. A. A., E. M. VENTINCIQUE \& A. D. BRESCOVIT, 2007. Effects of forest fragmentation on four Ctenus spider populations (Araneae: Ctenidae) in central Amazonia, Brazil. Studies on Neotropical Fauna and Environment 42(2): 137-144.

RICETTI, J. \& A. B. BONALDO, 2008. Diversidade e estimativas de riqueza de aranhas em quatro fitofisionomias na Serra do Cachimbo, Pará, Brasil. Iheringia, Série Zoologia 98(1): 88-99.

SCHARFF, N., J. A. CODDINGTON, C. E. GRISWOLD, G. HORMIGA \& PER DE PLACE BJØRN, 2003. When to quit? Estimating spider species richness in a northern European deciduos forest. Journal of Arachnology 31(2): 246-273.

SCHNITZER, S. A. \& W. P. CARSON, 2001. Treefall gaps and the maintenance of species diversity in a tropical forest. Ecology 82(4): 913-919.

SILVA, D. \&J. A. CODDINGTON, 1996. Spiders of Pakitza (Madre de Dios, Peru): species richness and notes on community structure. In: D. E. WILSON \& A. SANDOVAL (Eds.): The biodiversity of Pakitza and its environs: 241-299. Smithsonian Institution, Washington.

WILLETT, T. R., 2001. Spiders and other arthropods as indicators in old-growth versus logged redwood stands. Restoration Ecology 9(4): 410-420.

ZAR, J. H., 1999. Biostatistical analysis: 1-662 + app. Prentice Hall, Englewood Cliffs, New Jersey City.

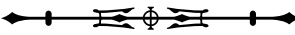


APÊNDICE. Lista de espécies de aranhas coletadas em Porto Urucu, Coari, Amazonas, Brasil, entre julho e novembro de 2006 , com as respectivas abundâncias totais em 33 clareiras amostradas.

(Continua)

\begin{tabular}{|c|c|c|}
\hline Família & Espécie & Exemplares \\
\hline \multirow{4}{*}{ Anapidae } & Anapis aff. saladito & 3 \\
\hline & Anapis castilla Platnick \& Shadab, 1978 & 1 \\
\hline & Anapisona aragua Platnick \& Shadab, 1979 & 14 \\
\hline & Pseudanapis sp.1 & 5 \\
\hline \multirow{11}{*}{ Anyphaenidae } & Anyphaenidae sp.1 & 2 \\
\hline & Anyphaenidae sp.2 & 1 \\
\hline & Anyphaenidae sp.3 & 1 \\
\hline & Isigonia limbata Simon, 1897 & 1 \\
\hline & Patrera sp.1 & 6 \\
\hline & Patrera sp.2 & 15 \\
\hline & Patrera sp.3 & 2 \\
\hline & Patrera sp.4 & 2 \\
\hline & Patrera sp.5 & 1 \\
\hline & Wulfila sp.1 & 3 \\
\hline & Wulfila sp.2 & 2 \\
\hline \multirow{20}{*}{ Araneidae } & Acacesia benigna Glueck, 1994 & 2 \\
\hline & Alpaida antonio Levi, 1988 & 11 \\
\hline & Alpaida delicata (Keyserling, 1892) & 90 \\
\hline & Alpaida guto Abrahim \& Bonaldo, 2008 & 29 \\
\hline & Alpaida hoffmani Levi, 1988 & 1 \\
\hline & Alpaida negro Levi, 1988 & 7 \\
\hline & Alpaida sp.1 & 7 \\
\hline & Alpaida sp.2 & 9 \\
\hline & Alpaida sp.3 & 6 \\
\hline & Alpaida sp.4 & 1 \\
\hline & Alpaida sp.5 & 11 \\
\hline & Alpaida sp.6 & 1 \\
\hline & Alpaida sp.7 & 1 \\
\hline & Alpaida sp.8 & 2 \\
\hline & Alpaida sp.9 & 4 \\
\hline & Alpaida sp.10 & 4 \\
\hline & Alpaida sp.11 & 1 \\
\hline & Alpaida truncata (Keyserling, 1865) & 2 \\
\hline & Amazonepeira callaria (Levi, 1991) & 23 \\
\hline & Amazonepeira herrera Levi, 1989 & 15 \\
\hline
\end{tabular}

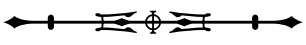




\begin{tabular}{|c|c|c|}
\hline Família & Espécie & Exemplares \\
\hline \multirow{36}{*}{ Araneidae } & Amazonepeira masaka Levi, 1994 & 13 \\
\hline & Araneidae sp.1 & 3 \\
\hline & Araneidae sp.2 & 1 \\
\hline & Araneidae sp.3 & 1 \\
\hline & Araneidae sp.4 & 1 \\
\hline & Araneus guttatus (Keyserling, 1865) & 1 \\
\hline & Bertrana elinguis (Keyserling, 1883) & 13 \\
\hline & cf. Alpaida delicate & 1 \\
\hline & Chaetacis cornuta (Taczanowski, 1873) & 9 \\
\hline & Chaetacis sp.1 & 2 \\
\hline & Cyclosa caroli (Hentz, 1850) & 2 \\
\hline & Cyclosa diversa (O. P.-Cambridge, 1894) & 1 \\
\hline & Cyclosa sp.1 & 28 \\
\hline & Cyclosa sp.2 & 9 \\
\hline & Cyclosa sp.3 & 2 \\
\hline & Cyclosa tapetifaciens Hingston, 1932 & 1 \\
\hline & Enacrossoma sp.1 & 1 \\
\hline & Eriophora fulliginea (C. L. Koch, 1838) & 2 \\
\hline & Eustala sp.1 & 3 \\
\hline & Eustala sp.2 & 16 \\
\hline & Eustala sp.3 & 19 \\
\hline & Eustala sp.4 & 1 \\
\hline & Eustala sp.5 & 2 \\
\hline & Eustala sp.6 & 4 \\
\hline & Eustala sp.7 & 4 \\
\hline & Eustala sp.8 & 3 \\
\hline & Eustala sp.9 & 3 \\
\hline & Eustala sp.10 & 3 \\
\hline & Eustala sp.11 & 1 \\
\hline & Hingstepeira foliscens (Hingston, 1932) & 2 \\
\hline & Hypognatha aff. deplanata & 6 \\
\hline & Kaira altiventer O. P.-Cambridge, 1889 & 1 \\
\hline & Mangora keduc Levi, 2007 & 4 \\
\hline & Mangora aff. acre & 69 \\
\hline & Mangora barba Levi, 2007 & 15 \\
\hline & Mangora dianasilvae Levi, 2007 & 29 \\
\hline
\end{tabular}




\begin{tabular}{|c|c|c|}
\hline Família & Espécie & Exemplares \\
\hline \multirow{37}{*}{ Araneidae } & Mangora mathani Simon, 1895 & 29 \\
\hline & Mangora moyobamba Levi, 2007 & 4 \\
\hline & Mangora novempupillata Mello-Leitão, 1940 & 1 \\
\hline & Mangora sp.1 & 1 \\
\hline & Mangora sp.2 & 3 \\
\hline & Mangora sp.3 & 1 \\
\hline & Manogea porracea (C. L. Koch, 1838) & 19 \\
\hline & Metazygia castaneoscutata (Simon, 1895) & 20 \\
\hline & Metazygia sendero Levi, 1995 & 1 \\
\hline & Metazygia sp.1 & 1 \\
\hline & Metazygia sp.2 & 1 \\
\hline & Metazygia tapa Levi, 1995 & 6 \\
\hline & Metazygia uma Levi, 1995 & 3 \\
\hline & Micrathena acuta (Walckenaer, 1842) & 4 \\
\hline & Micrathena clypeata (Walckenaer, 1805) & 35 \\
\hline & Micrathena coca Levi, 1985 & 3 \\
\hline & Micrathena embira Levi, 1985 & 1 \\
\hline & Micrathena exlinae Levi, 1985 & 7 \\
\hline & Micrathena kirbyi (Perty, 1833) & 21 \\
\hline & Micrathena miles Simon, 1895 & 13 \\
\hline & Micrathena pungens (Walckenaer, 1842) & 3 \\
\hline & Micrathena sp.1 & 1 \\
\hline & Micrathena sp.2 & 4 \\
\hline & Micrathena sp.3 & 1 \\
\hline & Micrathena sp.4 & 2 \\
\hline & Micrathena triangularispinosa (De Geer, 1778) & 34 \\
\hline & Micrathena vigorsi (Perty, 1833) & 3 \\
\hline & Micrepeira fowleri Levi, 1995 & 11 \\
\hline & Micrepeira hoeferi Levi, 1995 & 2 \\
\hline & Ocrepeira covillei Levi, 1993 & 14 \\
\hline & Parawixia hypocrita (O. P.-Cambridge, 1889) & 1 \\
\hline & Parawixia kochi (Taczanowski, 1873) & 21 \\
\hline & Parawixia ouro Levi, 1992 & 1 \\
\hline & Parawixia sp.1 & 8 \\
\hline & Scoloderus tuberculifer (O. P.-Cambridge, 1889) & 2 \\
\hline & Spilasma duodecimguttata (Keyserling, 1879) & 11 \\
\hline & Taczanowskia striata Keyserling, 1879 & 1 \\
\hline
\end{tabular}


APÊNDICE.

(Continua)

\begin{tabular}{|c|c|c|}
\hline Família & Espécie & Exemplares \\
\hline \multirow{9}{*}{ Araneidae } & Testudinaria aff. gravatai & 1 \\
\hline & Testudinaria quadripunctata Taczanowski, 1879 & 9 \\
\hline & Verrucosa sp.1 & 1 \\
\hline & Wagneriana hassleri Levi, 1991 & 4 \\
\hline & Wagneriana lechuza Levi, 1991 & 15 \\
\hline & Wagneriana roraima Levi, 1991 & 2 \\
\hline & Wagneriana silvae Levi, 1991 & 6 \\
\hline & Wagneriana sp.1 & 1 \\
\hline & Xylethrus superbus Simon, 1895 & 4 \\
\hline Caponiidae & Nops sp.1 & 3 \\
\hline \multirow{2}{*}{ Clubionidae } & Elaver sp.1 & 6 \\
\hline & Elaver sp.2 & 1 \\
\hline \multirow{25}{*}{ Corinnidae } & Abapeba sp.1 & 1 \\
\hline & Apochinomma sp.1 & 1 \\
\hline & Castianeira sp.1 & 1 \\
\hline & Castianeira sp.2 & 1 \\
\hline & Castianeira sp.3 & 1 \\
\hline & Castianeira sp.4 & 1 \\
\hline & Castianeira sp.5 & 2 \\
\hline & Corinna aff. bicincta sp.1 & 25 \\
\hline & Corinna aff. bicincta sp.2 & 9 \\
\hline & Corinna aff. ducke sp.1 & 3 \\
\hline & Corinna aff. ducke sp.2 & 1 \\
\hline & Corinna aff. eresiformes sp.1 & 1 \\
\hline & Corinna aff. eresiformes sp. 2 & 2 \\
\hline & Corinna gr. aenea & 4 \\
\hline & Corinna gr. kochi sp.1 & 4 \\
\hline & Corinna gr. kochi sp.2 & 5 \\
\hline & Corinna gr. kochi sp.3 & 2 \\
\hline & Corinna gr. kochi sp.4 & 2 \\
\hline & Corinna gr. kochi sp.5 & 1 \\
\hline & Corinninae sp.1 & 2 \\
\hline & Corinninae sp. 2 & 1 \\
\hline & Falconina sp.1 & 1 \\
\hline & Methesis semirufa Simon, 1896 & 2 \\
\hline & Myrmecium aff. dacetoniforme & 17 \\
\hline & Myrmecium sp.1 & 13 \\
\hline
\end{tabular}




\begin{tabular}{|c|c|c|}
\hline Família & Espécie & Exemplares \\
\hline \multirow{19}{*}{ Corinnidae } & Myrmecium sp.2 & 1 \\
\hline & Myrmecium sp.3 & 1 \\
\hline & Myrmecium sp.4 & 1 \\
\hline & Myrmecium sp.5 & 6 \\
\hline & Myrmecotypus sp.1 & 1 \\
\hline & Parachemmis aff. hassleri & 5 \\
\hline & Parachemmis sp.1 & 12 \\
\hline & Parachemmis sp.2 & 2 \\
\hline & Simonestus sp.1 & 1 \\
\hline & Simonestus sp.2 & 1 \\
\hline & Simonestus sp.3 & 1 \\
\hline & Sphecotypus sp.1 & 1 \\
\hline & Stethorrhagus lupulus Simon, 1896 & 3 \\
\hline & Trachelas sp.1 & 10 \\
\hline & Trachelas sp.2 & 3 \\
\hline & Trachelas sp.3 & 1 \\
\hline & Trachelas sp.4 & 1 \\
\hline & Tupirinna sp.1 & 2 \\
\hline & Xeropigo perene De Souza \& Bonaldo, 2007 & 1 \\
\hline \multirow{18}{*}{ Ctenidae } & Ancylometes rufus (Walckenaer, 1837) & 22 \\
\hline & Ctenidae sp.1 & 10 \\
\hline & Ctenidae sp.2 & 5 \\
\hline & Ctenidae sp.3 & 4 \\
\hline & Ctenidae sp.4 & 1 \\
\hline & Ctenidae sp.6 & 1 \\
\hline & Ctenidae sp.7 & 1 \\
\hline & Ctenidae sp.8 & 1 \\
\hline & Ctenidae sp.9 & 1 \\
\hline & Ctenus amphora Mello-Leitão, 1930 & 64 \\
\hline & Ctenus crulsi Mello-Leitão, 1930 & 61 \\
\hline & Ctenus inaja Höfer, Brescovit \& Gasnier, 1994 & 1 \\
\hline & Ctenus sp.1 & 18 \\
\hline & Ctenus sp.2 & 5 \\
\hline & Ctenus sp.3 & 2 \\
\hline & Ctenus sp.4 & 1 \\
\hline & Ctenus villasboasi Mello-Leitão, 1949 & 2 \\
\hline & Cupiennius bimaculatus (Taczanowski, 1874) & 18 \\
\hline
\end{tabular}


APÊNDICE.

(Continua)

\begin{tabular}{|c|c|c|}
\hline Família & Espécie & Exemplares \\
\hline \multirow{3}{*}{ Ctenidae } & Enoploctenus sp.1 & 5 \\
\hline & Gephyroctenus sp.1 & 6 \\
\hline & Phoneutria reidyi (F. O. P.-Cambridge, 1897) & 4 \\
\hline Deinopidae & Deinopis sp.1 & 24 \\
\hline \multirow{3}{*}{ Dipluridae } & Diplura sp.1 & 3 \\
\hline & Diplura sp.2 & 1 \\
\hline & Diplura sp.3 & 2 \\
\hline Drymusidae & Drymusa sp.1 & 1 \\
\hline \multirow{3}{*}{ Gnaphosidae } & Gnaphosidae sp.1 & 1 \\
\hline & Zimiromus aff. reichardti & 6 \\
\hline & Zimiromus sp.1 & 1 \\
\hline Hahniidae & Neohania ernsti (Simon, 1897) & 4 \\
\hline Hersilidae & Ypypuera crucifera (Vellard, 1924) & 1 \\
\hline \multirow{14}{*}{ Linyphiidae } & Linyphiidae sp.1 & 20 \\
\hline & Linyphiidae sp.2 & 29 \\
\hline & Linyphiidae sp.3 & 12 \\
\hline & Linyphiidae sp.4 & 6 \\
\hline & Linyphiidae sp.5 & 5 \\
\hline & Linyphiidae sp.6 & 6 \\
\hline & Linyphiidae sp.7 & 7 \\
\hline & Linyphiidae sp.8 & 2 \\
\hline & Linyphiidae sp.9 & 1 \\
\hline & Linyphiidae sp.10 & 1 \\
\hline & Linyphiidae sp.11 & 1 \\
\hline & Linyphiidae sp.12 & 1 \\
\hline & Mermessus conjunctus (Millidge, 1991) & 1 \\
\hline & Sphecozone crassa (Millidge, 1991) & 1 \\
\hline \multirow{2}{*}{ Lycosidae } & Aglaoctenus lagotis (Holmberg, 1876) & 1 \\
\hline & Lycosidae sp.1 & 1 \\
\hline \multirow{8}{*}{ Mimetidae } & Arocha sp.1 & 7 \\
\hline & Ero sp.1 & 2 \\
\hline & Ero sp.2 & 1 \\
\hline & Ero sp.3 & 6 \\
\hline & Ero sp.4 & 6 \\
\hline & Ero sp.5 & 2 \\
\hline & Gelanor heraldicus Petrunkevitch, 1925 & 2 \\
\hline & Gelanor mabelae Chickering, 1947 & 1 \\
\hline
\end{tabular}

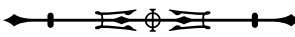




\begin{tabular}{|c|c|c|}
\hline Família & Espécie & Exemplares \\
\hline \multirow{3}{*}{ Mimetidae } & Gelanor sp.1 & 4 \\
\hline & Mimetinae sp.1 & 3 \\
\hline & Mimetinae sp.2 & 2 \\
\hline \multirow{3}{*}{ Mysmenidae } & Mysmenidae sp.1 & 1 \\
\hline & Mysmenidae sp.2 & 1 \\
\hline & Mysmenidae sp.3 & 1 \\
\hline Nephilidae & Nephila clavipes (Linnaeus, 1767) & 4 \\
\hline Ochyroceratidae & Ochyroceratidae sp.1 & \\
\hline \multirow{21}{*}{ Oonopidae } & aff. Triaeris sp.1 & 2 \\
\hline & Dysderina sp.1 & 6 \\
\hline & Dysderina sp.2 & 2 \\
\hline & Dysderina sp.3 & 2 \\
\hline & Dysderina sp.4 & 5 \\
\hline & Gamasomorphinae sp.1 & 6 \\
\hline & Neoxyphinus sp.1 & 3 \\
\hline & Neoxyphinus sp.2 & 8 \\
\hline & Neoxyphinus sp.3 & 1 \\
\hline & Oonopidae gen. 1 sp.1 & 5 \\
\hline & Oonopidae gen. 2 sp.1 & 1 \\
\hline & Oonopidae gen. 2 sp.2 & 1 \\
\hline & Oonopidae gen. 3 sp.1 & 1 \\
\hline & Oonopidae gen. 3 sp.2 & 1 \\
\hline & Oonopidae gen. 3 sp.3 & 1 \\
\hline & Oonopidae gen. 3 sp.4 & 1 \\
\hline & Oonopidae gen. 3 sp.5 & 1 \\
\hline & Oonopidae gen. 3 sp. 6 & 1 \\
\hline & Oonopidae gen. 4 sp.1 & 1 \\
\hline & Oonopidae gen. 5 sp.1 & 1 \\
\hline & Simonoonops sp.1 & 3 \\
\hline \multirow{7}{*}{ Oxyopidae } & Oxyopes hemorrhous Mello-Leitão, 1929 & 1 \\
\hline & Oxyopes holmbergi Soares \& Camargo, 1948 & 41 \\
\hline & Oxyopes incertus Mello-Leitão, 1929 & 47 \\
\hline & Oxyopes sp.1 & 1 \\
\hline & Oxyopes sp.7 & 3 \\
\hline & Schaenicoscelis sp.n.2 & 1 \\
\hline & Tapinillus longipes (Taczanowski, 1872) & 1 \\
\hline Pholcidae & Carapoia ocaina Huber, 2000 & 24 \\
\hline
\end{tabular}

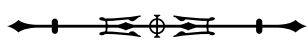




\begin{tabular}{|c|c|c|}
\hline Família & Espécie & Exemplares \\
\hline \multirow{10}{*}{ Pholcidae } & Ibotyporanga sp.1 & 1 \\
\hline & Litoporus dimona Huber, 2000 & 6 \\
\hline & Litoporus sp.1 & 3 \\
\hline & Mesabolivar aurantiacus (Mello-Leitão, 1930) & 20 \\
\hline & Mesabolivar sp.1 & 42 \\
\hline & Metagonia beni Huber, 2000 & 8 \\
\hline & Metagonia taruma Huber, 2000 & 75 \\
\hline & Otavaloa sp.1 & 1 \\
\hline & Pholcidae sp.1 & 1 \\
\hline & Pholcidae sp.2 & 1 \\
\hline \multirow{5}{*}{ Pisauridae } & Architis cymatilis Carico, 1981 & 1 \\
\hline & Architis tenuis Simon, 1898 & 25 \\
\hline & Thaumasia argenteonotata (Simon, 1898) & 1 \\
\hline & Thaumasia sp.1 & 3 \\
\hline & Thaumasia sp.2 & 1 \\
\hline \multirow{22}{*}{ Salticidae } & Amycinae sp.1 & 2 \\
\hline & Amycinae sp.3 & 1 \\
\hline & Cotinusa sp.1 & 26 \\
\hline & Cylistella sp.1 & 5 \\
\hline & Cylistella sp.2 & 1 \\
\hline & Euophryinae sp.1 & 2 \\
\hline & Euophryinae sp.2 & 8 \\
\hline & Euophryinae sp.3 & 3 \\
\hline & Euophryinae sp.4 & 7 \\
\hline & Euophryinae sp. 5 & 6 \\
\hline & Euophryinae sp.6 & 4 \\
\hline & Euophryinae sp.7 & 3 \\
\hline & Euophryinae sp.8 & 5 \\
\hline & Euophryinae sp.9 & 5 \\
\hline & Euophryinae sp.10 & 19 \\
\hline & Euophryinae sp.11 & 1 \\
\hline & Euophryinae sp.12 & 1 \\
\hline & Euophryinae sp.13 & 1 \\
\hline & Fluda sp.1 & 8 \\
\hline & Fluda sp.2 & 5 \\
\hline & Hypaeus miles Simon, 1900 & 5 \\
\hline & Hyраеus sp.1 & 36 \\
\hline
\end{tabular}




\begin{tabular}{|c|c|c|}
\hline Família & Espécie & Exemplares \\
\hline \multirow{37}{*}{ Salticidae } & Hypaeus sp.2 & 9 \\
\hline & Hypaeus sp.3 & 21 \\
\hline & Hypaeus sp.4 & 7 \\
\hline & Itata sp.1 & 4 \\
\hline & Kalcerrytus sp.1 & 1 \\
\hline & Lyssomanes nigropictus Peckham \& Wheeler, 1889 & 16 \\
\hline & Lyssomanes sp.1 & 15 \\
\hline & Lyssomanes sp.2 & 1 \\
\hline & Lyssomanes sp.3 & 1 \\
\hline & Lyssomanes tenuis Peckham \& Wheeler, 1889 & 15 \\
\hline & Martella sp.1 & 1 \\
\hline & Noegus sp.1 & 3 \\
\hline & Noegus sp.2 & 28 \\
\hline & Noegus sp.3 & 12 \\
\hline & Noegus sp.4 & 3 \\
\hline & Noegus sp.5 & 12 \\
\hline & Pachomius sp.1 & 1 \\
\hline & Pachomius sp.2 & 3 \\
\hline & Pachomius sp. 3 & 1 \\
\hline & Phiale sp.1 & 1 \\
\hline & Psecas sp.1 & 4 \\
\hline & Romitia sp.1 & 2 \\
\hline & Romitia sp.2 & 2 \\
\hline & Romitia sp.3 & 1 \\
\hline & Salticidae sp.1 & 9 \\
\hline & Salticidae sp.2 & 5 \\
\hline & Salticidae sp.3 & 4 \\
\hline & Salticidae sp.4 & 7 \\
\hline & Salticidae sp.5 & 3 \\
\hline & Salticidae sp.6 & 3 \\
\hline & Salticidae sp.7 & 2 \\
\hline & Salticidae sp. 8 & 3 \\
\hline & Salticidae sp.9 & 2 \\
\hline & Salticidae sp.10 & 2 \\
\hline & Salticidae sp.11 & 2 \\
\hline & Salticidae sp.12 & 2 \\
\hline & Salticidae sp.13 & 2 \\
\hline
\end{tabular}




\begin{tabular}{|c|c|c|}
\hline Família & Espécie & Exemplares \\
\hline \multirow{22}{*}{ Salticidae } & Salticidae sp.14 & 2 \\
\hline & Salticidae sp.15 & 1 \\
\hline & Salticidae sp.16 & 1 \\
\hline & Salticidae sp.17 & 1 \\
\hline & Salticidae sp.18 & 1 \\
\hline & Salticidae sp.19 & 1 \\
\hline & Salticidae sp.20 & 1 \\
\hline & Salticidae sp.21 & 1 \\
\hline & Salticidae sp.22 & 1 \\
\hline & Salticidae sp.23 & 1 \\
\hline & Salticidae sp.24 & 1 \\
\hline & Salticidae sp.25 & 1 \\
\hline & Salticidae sp.26 & 1 \\
\hline & Salticidae sp.28 & 1 \\
\hline & Salticidae sp.29 & 1 \\
\hline & Sassacus sp.1 & 1 \\
\hline & Scopocira sp.1 & 3 \\
\hline & Scopocira sp.2 & 3 \\
\hline & Sidusa sp.1 & 11 \\
\hline & Sidusa sp.2 & 15 \\
\hline & Sidusa sp. 3 & 17 \\
\hline & Synemosyna sp.1 & 3 \\
\hline \multirow{2}{*}{ Scytodidae } & Scytodes auricula Rheims \& Brescovit, 2000 & 49 \\
\hline & Scytodes lineatipes Taczanowski, 187 & 3 \\
\hline \multirow{2}{*}{ Segestriidae } & Segestriidae sp.1 & 1 \\
\hline & Segestriidae sp.2 & 1 \\
\hline \multirow{7}{*}{ Selenopidae } & Selenopidae sp.1 & 1 \\
\hline & Selenopidae sp.2 & 1 \\
\hline & Selenopidae sp.3 & 1 \\
\hline & Selenopidae sp.4 & 1 \\
\hline & Selenopidae sp. 5 & 1 \\
\hline & Selenopidae sp.6 & 1 \\
\hline & Selenopidae sp.7 & 2 \\
\hline \multirow{3}{*}{ Senoculidae } & Senoculus sp.1 & 7 \\
\hline & Senoculus sp.2 & 6 \\
\hline & Senoculus sp. 3 & 1 \\
\hline Sparassidae & Macrinus succineus Simon, 1887 & 1 \\
\hline
\end{tabular}




\begin{tabular}{|c|c|c|}
\hline Família & Espécie & Exemplares \\
\hline \multirow{13}{*}{ Sparassidae } & Olios keyserlingi (Simon, 1880) & 2 \\
\hline & Olios pictitarsis (Simon, 1880) & 2 \\
\hline & Olios velox (Simon, 1880) & 3 \\
\hline & Pseudoparianthis ravida Simon, 1897 & 20 \\
\hline & Sparassidae sp.1 & 1 \\
\hline & Sparassidae sp.2 & 1 \\
\hline & Sparassidae sp.3 & 1 \\
\hline & Sparassidae sp.4 & 5 \\
\hline & Sparassidae sp.5 & 1 \\
\hline & Sparassidae sp. 6 & 1 \\
\hline & Sparassidae sp.7 & 1 \\
\hline & Sparassidae sp. 8 & 1 \\
\hline & Sparianthinae sp.1 & 2 \\
\hline \multirow{3}{*}{ Symphytognathidae } & Symphytognathidae sp.1 & 1 \\
\hline & Symphytognathidae sp.2 & 1 \\
\hline & Symphytognathidae sp.3 & 1 \\
\hline \multirow{3}{*}{ Synotaxidae } & Synotaxus siolii Santos \& Rheims, 2005 & 1 \\
\hline & Synotaxus sp.n.2 & 2 \\
\hline & Synotaxus waiwai Agnarsson, 2003 & 1 \\
\hline \multirow{18}{*}{ Tetragnathidae } & Chrysometa sp.1 & 1 \\
\hline & Chrysometa sp.4 & 2 \\
\hline & Dolichognatha sp.1 & 2 \\
\hline & Leucauge sp.1 & 13 \\
\hline & Leucauge sp.2 & 11 \\
\hline & Leucauge sp.3 & 6 \\
\hline & Leucauge sp.4 & 3 \\
\hline & Leucauge sp. 5 & 5 \\
\hline & Leucauge sp.6 & 4 \\
\hline & Leucauge sp.7 & 3 \\
\hline & Leucauge sp. 8 & 2 \\
\hline & Leucauge sp.9 & 1 \\
\hline & Leucauge sp.10 & 1 \\
\hline & Leucauge sp.11 & 1 \\
\hline & Leucauge sp.12 & 1 \\
\hline & Leucauge sp.13 & 1 \\
\hline & Tetragnatha sp.1 & 1 \\
\hline & Tetragnatha sp.2 & 1 \\
\hline
\end{tabular}


APÊNDICE.

(Continua)

\begin{tabular}{|c|c|c|}
\hline Família & Espécie & Exemplares \\
\hline \multirow{2}{*}{ Tetragnathidae } & Tetragnatha sp.3 & 1 \\
\hline & Tetragnathidae sp.1 & 1 \\
\hline \multirow{5}{*}{ Theraphosidae } & aff. Proshapalopus sp.1 & 2 \\
\hline & Cyriocosmus aff. ritae & 1 \\
\hline & Ischonocolinae sp.1 & 1 \\
\hline & Pamphobeteus sp.1 & 2 \\
\hline & Theraphosinae sp.3 & 1 \\
\hline \multirow{30}{*}{ Theridiidae } & Achaearanea hieroglyphica (Mello-Leitão, 1940) & 9 \\
\hline & Achaearanea sp.1 & 1 \\
\hline & Achaearanea sp.2 & 8 \\
\hline & Achaearanea sp.3 & 1 \\
\hline & Achaearanea sp.4 & 1 \\
\hline & Achaearanea tingo Levi, 1963 & 9 \\
\hline & Achaearenea trapezoidalis (Taczanowski, 1873) & 3 \\
\hline & Achaeranea nigrovittata (Keyserling, 1884) & 1 \\
\hline & Ameridion sp.1 & 3 \\
\hline & Ameridion sp.2 & 1 \\
\hline & Ameridion sp.3 & 1 \\
\hline & Ameridion sp.4 & 1 \\
\hline & Anelosimus eximius (Keyserling, 1884) & 2 \\
\hline & Ariamnes attenuatus O. P.-Cambridge, 1881 & 2 \\
\hline & Ariamnes sp.1 & 5 \\
\hline & Cerocida ducke Marques \& Buckup, 1989 & 1 \\
\hline & Cerocida strigosa Simon, 1894 & 1 \\
\hline & cf. Ariamnes attenuatus & 2 \\
\hline & cf. Dipoena alta & 2 \\
\hline & cf. Dipoena augara & 2 \\
\hline & cf. Dipoena militaris & 2 \\
\hline & cf. Episinus salobrensis & 1 \\
\hline & Chrosiothes sp.10 & 12 \\
\hline & Chrosiothes sp.11 & 10 \\
\hline & Chrosiothes sp.12 & 1 \\
\hline & Chrysso sp.18 & 21 \\
\hline & Coleosoma acutiventer (Keyserling, 1884) & 2 \\
\hline & Cryptachaea pydanieli (Buckup \& Marques, 1991) & 2 \\
\hline & Cryptachaea schneirlai (Levi, 1959) & 9 \\
\hline & Cryptachaea uviana (Levi, 1963) & 5 \\
\hline
\end{tabular}

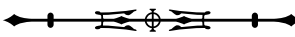




\begin{tabular}{|c|c|c|}
\hline Família & Espécie & Exemplares \\
\hline \multirow{37}{*}{ Theridiidae } & Dipoena atlantica Chickering, 1943 & 26 \\
\hline & Dipoena bryantae Chickering, 1943 & 1 \\
\hline & Dipoena cordiformis Keyserling, 1886 & 1 \\
\hline & Dipoena duodecimpunctata Chickering, 1943 & 3 \\
\hline & Dipoena hortoni Chickering, 1943 & 10 \\
\hline & Dipoena kuyumini Levi, 1963 & 1 \\
\hline & Dipoena militaris Chickering, 1943 & 10 \\
\hline & Dipoena olivenca Levi, 1963 & 4 \\
\hline & Dipoena rubella (Keyserling, 1884) & 4 \\
\hline & Dipoena sp.1 & 1 \\
\hline & Dipoena sp.2 & 1 \\
\hline & Dipoena sp.3 & 2 \\
\hline & Dipoena sp.4 & 7 \\
\hline & Dipoena sp.5 & 1 \\
\hline & Dipoena sp.6 & 5 \\
\hline & Dipoena sp.7 & 2 \\
\hline & Dipoena sp.8 & 6 \\
\hline & Dipoena sp.9 & 8 \\
\hline & Dipoena sp.10 & 1 \\
\hline & Dipoena sp.11 & 2 \\
\hline & Dipoena sp.12 & 1 \\
\hline & Dipoena sp.13 & 3 \\
\hline & Dipoena tingo Levi, 1963 & 2 \\
\hline & Dipoena tiro Levi, 1963 & 2 \\
\hline & Echinotheridion levii Ramírez \& González, 1999 & 1 \\
\hline & Echinotheridion utibile (Keyserling, 1884) & 2 \\
\hline & Episinus juarezi Levi, 1955 & 4 \\
\hline & Episinus malachinus (Simon, 1895) & 11 \\
\hline & Episinus nebulosus (Simon, 1895) & 84 \\
\hline & Episinus salobrensis (Simon, 1895) & 275 \\
\hline & Episinus sp.1 & 41 \\
\hline & Episinus sp.2 & 3 \\
\hline & Episinus sp.3 & 2 \\
\hline & Episinus sp.4 & 4 \\
\hline & Faiditus convolutus (Exline \& Levi, 1962) & 28 \\
\hline & Faiditus sp.1 & 1 \\
\hline & Faiditus sp.2 & 9 \\
\hline
\end{tabular}

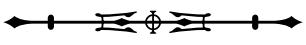




\begin{tabular}{|c|c|c|}
\hline Família & Espécie & Exemplares \\
\hline \multirow{37}{*}{ Theridiidae } & Faiditus sp.3 & 1 \\
\hline & Faiditus sp.4 & 1 \\
\hline & Faiditus sp.5 & 1 \\
\hline & Faiditus sp.6 & 1 \\
\hline & Faiditus sp.7 & 1 \\
\hline & Faiditus sp.8 & 17 \\
\hline & Faiditus sp.9 & 1 \\
\hline & Faiditus sp.10 & 1 \\
\hline & Hadrotarsinae sp.1 & 1 \\
\hline & Hadrotarsinae sp.2 & 1 \\
\hline & Helvibis longicauda Keyserling, 1891 & 13 \\
\hline & Helvibis sp.1 & 1 \\
\hline & Helvibis sp.2 & 12 \\
\hline & Helvibis sp.3 & 4 \\
\hline & Hentziectypus rafaeli (Buckup \& Marques, 1991) & 8 \\
\hline & Lasaeola donaldi (Chickering, 1943) & 11 \\
\hline & Phoroncidia aff. moyobamba & 11 \\
\hline & Phycosoma sp.1 & 4 \\
\hline & Rhomphaea sp.1 & 3 \\
\hline & Rhomphaea sp.2 & 9 \\
\hline & Spintharinae sp.1 & 4 \\
\hline & Spintharus flavidus Hentz, 1850 & 2 \\
\hline & Stemmops sp.5 & 2 \\
\hline & Styposis sp.4 & 5 \\
\hline & Styposis sp. 5 & 3 \\
\hline & Styposis sp.6 & 1 \\
\hline & Styposis sp.7 & 2 \\
\hline & Styposis sp.8 & 1 \\
\hline & Tekellina sp.1 & 1 \\
\hline & Theridiinae sp.1 & 2 \\
\hline & Theridion incertissimum (Caporiacco, 1954) & 2 \\
\hline & Theridion sp.1 & 1 \\
\hline & Theridion sp.2 & 1 \\
\hline & Theridion sp.3 & 1 \\
\hline & Theridion sp.4 & 1 \\
\hline & Theridion sp.5 & 1 \\
\hline & Theridion sp.6 & 1 \\
\hline
\end{tabular}




\begin{tabular}{|c|c|c|}
\hline Família & Espécie & Exemplares \\
\hline \multirow{12}{*}{ Theridiidae } & Theridion sp.7 & 1 \\
\hline & Theridion sp.8 & 1 \\
\hline & Theridion sp.9 & 1 \\
\hline & Theridion sp.10 & 1 \\
\hline & Thwaitesia bracteata (Exline, 1950) & 23 \\
\hline & Thymoites sp.1 & 21 \\
\hline & Thymoites sp. 2 & 3 \\
\hline & Thymoites sp. 3 & 2 \\
\hline & Thymoites sp.4 & 3 \\
\hline & Thymoites sp. 5 & 2 \\
\hline & Thymoites sp.6 & 1 \\
\hline & Thymoites sp.7 & 5 \\
\hline \multirow{18}{*}{ Theridiosomatidae } & Chthonos sp.1 & 6 \\
\hline & Naatlo sp.1 & 34 \\
\hline & Naatlo sp.2 & 5 \\
\hline & Naatlo sp.3 & 4 \\
\hline & Theridiosomatidae sp.1 & 1 \\
\hline & Theridiosomatidae sp.2 & 1 \\
\hline & Theridiosomatidae sp. 3 & 1 \\
\hline & Theridiosomatidae sp.4 & 6 \\
\hline & Theridiosomatidae sp.5 & 2 \\
\hline & Theridiosomatidae sp. 6 & 7 \\
\hline & Theridiosomatidae sp.7 & 1 \\
\hline & Theridiosomatidae sp. 8 & 1 \\
\hline & Theridiosomatidae sp.9 & 1 \\
\hline & Theridiosomatidae sp. 10 & 2 \\
\hline & Theridiosomatidae sp.11 & 11 \\
\hline & Theridiosomatidae sp. 12 & 2 \\
\hline & Theridiosomatidae sp.13 & 1 \\
\hline & Theridiosomatidae sp.14 & 1 \\
\hline \multirow{7}{*}{ Thomisidae } & Acentroscelus sp.1 & 1 \\
\hline & Aphantochilus rogersi O. P.-Cambridge, 1870 & 1 \\
\hline & Bucranium taurifrons O. P.-Cambridge, 1881 & 4 \\
\hline & Epicadus sp.1 & 3 \\
\hline & Onoculus echinatus (Taczanowski, 1872) & 17 \\
\hline & Onoculus sp.n. & 1 \\
\hline & Stephanopoides simoni Keyserling, 1880 & 8 \\
\hline
\end{tabular}

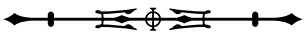




\begin{tabular}{|c|c|c|}
\hline Família & Espécie & Exemplares \\
\hline \multirow{21}{*}{ Thomisidae } & Strophius cf. fidelis & 1 \\
\hline & Strophius fidelis Mello-Leitão, 1929 & 2 \\
\hline & Strophius nigricans Keyserling, 1880 & 4 \\
\hline & Strophius sp.1 & 1 \\
\hline & Synema sp.1 & 1 \\
\hline & Thomisinae sp.1 & 5 \\
\hline & Thomisinae sp.2 & 17 \\
\hline & Thomisinae sp.3 & 5 \\
\hline & Thomisinae sp.4 & 2 \\
\hline & Thomisinae sp.5 & 2 \\
\hline & Tmarus sp. & 1 \\
\hline & Tmarus sp.1 & 2 \\
\hline & Tmarus sp.2 & 2 \\
\hline & Tmarus sp.3 & 1 \\
\hline & Tmarus sp.4 & 1 \\
\hline & Tmarus sp.5 & 2 \\
\hline & Tmarus sp.6 & 1 \\
\hline & Tobias cf. gradiens & 1 \\
\hline & Tobias sp.1 & 22 \\
\hline & Tobias sp.2 & 11 \\
\hline & Tobias sp.3 & 1 \\
\hline \multirow{7}{*}{ Trechaleidae } & Dossenus marginatus Simon, 1898 & 14 \\
\hline & Rhoicinus sp.n. & 7 \\
\hline & Rhoicinus urucu Brescovit \& Oliveira, 1994 & 7 \\
\hline & Syntrechalea adis Carico, 2008 & 1 \\
\hline & Syntrechalea caporiacco Carico, 2008 & 2 \\
\hline & Syntrechalea syntrechaloides (Mello-Leitão, 1941) & 5 \\
\hline & Syntrechalea tenuis F. O. P.-Cambridge, 1902 & 1 \\
\hline \multirow{9}{*}{ Uloboridae } & aff. Philoponella sp.1 & 17 \\
\hline & Miagrammopes sp.1 & 1 \\
\hline & Miagrammopes sp.2 & 4 \\
\hline & Miagrammopes sp.3 & 5 \\
\hline & Miagrammopes sp.4 & 4 \\
\hline & Miagrammopes sp.5 & 11 \\
\hline & Miagrammopes sp.6 & 11 \\
\hline & Miagrammopes sp.7 & 3 \\
\hline & Miagrammopes sp.8 & 1 \\
\hline
\end{tabular}




\begin{tabular}{|c|c|c|}
\hline Família & Espécie & Exemplares \\
\hline \multirow{27}{*}{ Uloboridae } & Miagrammopes sp. 9 & 3 \\
\hline & Miagrammopes sp.10 & 2 \\
\hline & Miagrammopes sp.11 & 2 \\
\hline & Miagrammopes sp.12 & 2 \\
\hline & Philoponella sp.1 & 24 \\
\hline & Philoponella sp.2 & 16 \\
\hline & Philoponella sp.3 & 5 \\
\hline & Philoponella sp.4 & 1 \\
\hline & Philoponella sp.5 & 1 \\
\hline & Philoponella sp.6 & 1 \\
\hline & Philoponella sp.7 & 9 \\
\hline & Philoponella sp.8 & 2 \\
\hline & Philoponella sp.9 & 6 \\
\hline & Philoponella sp.10 & 4 \\
\hline & Philoponella sp.11 & 3 \\
\hline & Philoponella sp.12 & 3 \\
\hline & Philoponella sp.13 & 3 \\
\hline & Philoponella sp.14 & 2 \\
\hline & Philoponella sp.15 & 1 \\
\hline & Philoponella sp.16 & 1 \\
\hline & Zosis sp.1 & 2 \\
\hline & Zosis sp.2 & 7 \\
\hline & Zosis sp. 3 & 2 \\
\hline & Zosis sp.4 & 1 \\
\hline & Zosis sp. 5 & 3 \\
\hline & Zosis sp.6 & 2 \\
\hline & Zosis sp.7 & 4 \\
\hline \multirow{2}{*}{ Zodariidae } & Epicratinus amazonicus Jocqué \& Baert, 2005 & 8 \\
\hline & Tenedos hoeferi Jocqué \& Baert, 2002 & 2 \\
\hline
\end{tabular}

